\title{
Skin perfusion pressure for the prediction of wound healing in critical limb ischemia: a meta-analysis
}

Xuanliang Pan, Chuangang You, Guoxian Chen, Huawei Shao, Chunmao Han, Lizhu Zhi

Department of Burns and Wound Center, The Second Affiliated Hospital of Zhejiang University School of Medicine, Hangzhou, China

Submitted: 26 November 2015

Accepted: 24 March 2016

Arch Med Sci 2018; 14, 3: 481-487

DOI: https://doi.org/10.5114/aoms.2016.62220

Copyright $\odot 2016$ Termedia \& Banach

\section{Abstract}

Introduction: The purpose of this meta-analysis was to determine the value and efficacy of skin perfusion pressure (SPP) for the prediction of wound healing in patients with critical limb ischemia.

Material and methods: Medline, Cochrane, EMBASE, and Google Scholar databases were searched from inception until December 31, 2014 using combinations of the following keywords: skin perfusion pressure, limb ischemia, wound healing, prediction. Randomized controlled trials, 2-arm prospective studies, and retrospective studies that measured SPP in patients with limb ischemia were included. The outcome was the sensitivity and specificity of SPP for the prediction of wound healing.

Results: Five studies were included in the meta-analysis. The mean patient age ranged from 62.2 to 71.5 years, and the majority were male. The pooled sensitivity of SPP for the prediction of wound healing was $79.9 \%$ using $30 \mathrm{~mm} \mathrm{Hg}$ as the cut-off, $67.1 \%$ using $40 \mathrm{~mm} \mathrm{Hg}$, and $76.1 \%$ for all included studies (95\% Cl: $73.9-84.9 \%, 55.8-76.8 \%$, and $70.7-80.8 \%$, respectively). The pooled specificity was $78.2 \%$ using $30 \mathrm{~mm} \mathrm{Hg}, 84.2 \%$ using $40 \mathrm{~mm} \mathrm{Hg}$, and $82.1 \%$ for all included studies $(95 \% \mathrm{Cl}$ : $61.5-89.0 \%, 74.0-90.9 \%, 73.7-$ $88.3 \%$, respectively).

Conclusions: Skin perfusion pressure can accurately predict wound healing in patients with critical limb ischemia.

Key words: amputation, limb ischemia, meta-analysis, skin perfusion pressure.

\section{Introduction}

Critical limb ischemia is characterized by chronic ischemic at-rest pain, ulcers, or gangrene in one or both lower limbs that is due to objectively proven arterial occlusive disease [1]. Once critical limb ischemia has occurred, amputation may be inevitable, although advances in gene therapy hold promise [2]. Limb ischemia affects blood flow to the skin, which is an important factor that affects wound healing, and is considered when determining if an amputation is necessary and at what level to perform the amputation [3]. Wound healing may also be affected by limb ischemia after an amputation has been performed [4].

Measurement of skin perfusion pressure (SPP) is an accurate and non-invasive method for evaluation of blood flow to impaired tissue [5]. Skin perfusion pressure is most commonly measured by laser Doppler $[6,7]$, though radioisotope clearance [8] and photoplethysmography

\author{
Corresponding author: \\ Xuanliang Pan \\ Department of Burns \\ and Wound Center \\ The Second \\ Affiliated Hospital \\ of Zhejiang University \\ School of Medicine \\ 88\# JieFang Road \\ Hangzhou \\ Zhejiang Province \\ 310009, China \\ Phone: +86 57187783661 \\ Fax: +86 57187784585 \\ E-mail: panxuanliang@zju. \\ edu.cn
}


have also been used [8]. Regardless of the method, the principle is the same. A cuff is inflated at the site of measurement, the pressure is slowly released, and the pressure at which the movement of red blood cells, washout of isotope, or the reappearance of pulsatile flux occurs is recorded as the SPP [6-8]. The pressure applied to the underlying skin at which blood flow ceases is defined as the SPP.

Unlike other methods of evaluating blood flow to ischemic tissue, SPP is not affected by calcified arteries [9]. Skin perfusion pressure has been shown to be an independent predictor of wound healing in patients with limb ischemia, and is useful for deciding between conservative therapy, revascularization, and the need for amputation as well as the level of amputation [5, 9]. There is, however, debate with respect to the cut-off point of SPP used for decision making, with $30 \mathrm{~mm} \mathrm{Hg}$ and $40 \mathrm{~mm} \mathrm{Hg}$ the most commonly used [9].

Thus the purpose of this study was to perform a meta-analysis to determine the sensitivity and specificity of SPP for the prediction of wound healing in patients with critical limb ischemia, and to examine the utility of 2 cut-off points, $30 \mathrm{~mm} \mathrm{Hg}$ and $40 \mathrm{~mm} \mathrm{Hg}$.

\section{Material and methods}

\section{Literature search strategy and study selection criteria}

This systematic review and meta-analysis was conducted in accordance with PRISMA guidelines [10]. Medline, Cochrane, EMBASE, and Google Scholar databases were searched from inception until December 31, 2014 using combinations of the keywords: skin perfusion pressure, limb ischemia, wound healing, prediction. Reference lists of relevant studies were hand-searched. Two independent reviewers performed database searches to identify potentially relevant articles, and article titles and abstracts were screened based on the inclusion and exclusion criteria. Where there was uncertainty regarding eligibility, a third reviewer was consulted and a decision arrived at by consensus.

Inclusion criteria for the meta-analysis were: 1) randomized controlled trials (RCTs), 2-arm prospective studies, and retrospective studies; 2) patients with limbs receiving conservative treatment, endovascular therapy, or amputation; 3) SPP was measured; 4) study reported quantitative outcome data. Studies other than RCTs were included in the analysis because studies examining this topic are limited. Letters, comments, editorials, case reports, proceedings, personal communications and studies in which no quantitative outcome data were reported were excluded.

\section{Data extraction}

The following information/data were extracted from studies that met the inclusion criteria: the name of the first author, year of publication, study design, number of participants in each group, participants' age and gender, follow-up period, number of involved limbs, and outcome data, i.e., the sensitivity and specificity of SPP for the prediction of wound healing.

\section{Quality assessment}

The QUADAS-2 instrument was used to assess the quality of the included studies [11]. Briefly, the QUADAS-2 consists of four key domains: patient selection, index test, reference standard, flow and timing. Each domain is assessed in terms of risk of bias and the first 3 in terms of concerns regarding applicability.

\section{Outcome measures and statistical analysis}

The outcome was the sensitivity and specificity of SPP for the prediction of wound healing in patients with critical limb ischemia. The sensitivity and specificity of SPP measurement were calculated from contingency tables based on the optimal cut-off for predicting would healing in the individual study. The pooled sensitivity and specificity among studies were calculated, and reported with 95\% confidence intervals (Cls). Pooled estimates were calculated for the studies overall, and for SPP cut-off points of $30 \mathrm{~mm} \mathrm{Hg}$ and $40 \mathrm{~mm} \mathrm{Hg}$. Heterogeneity among the studies was assessed by Cochran $Q$ and the $I^{2}$ statistic. For the $Q$ statistic, $p<0.10$ was considered to indicate significant heterogeneity. The $R$ statistic indicates the percentage of the observed between-study variability caused by heterogeneity, and a value $\geq 50 \%$ was considered to indicate significant heterogeneity. If significant heterogeneity existed between studies ( $Q$ statistic with $p<0.1$ or $p^{2}$ statistic $>50 \%$ ) [12, 13], a random-effects model (DerSimonian-Laird method) of analysis was performed [14]. Otherwise, a fixed-effects model was used (MantelHaenszel method). Sensitivity analysis was performed based on the leave-one-out approach. All statistical analyses were performed with Comprehensive Meta-Analysis statistical software, version 2.0 (Biostat, Englewood, NJ, USA).

\section{Results}

\section{Study characteristics}

A flow diagram of study selection is shown in Figure 1. A total of 130 studies were identified in the database search. Of these, 109 non-relevant studies were excluded as they did not meet the inclusion/exclusion criteria based on a review of 


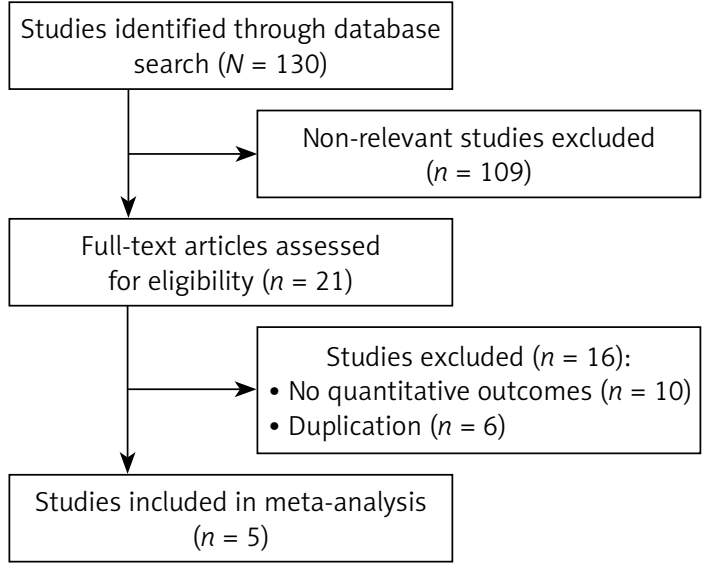

Figure 1. PRISMA flow diagram of study selection

the title and abstract, and 21 full text articles assessed for eligibility. Sixteen studies were excluded (no quantitative outcomes $n=10$, duplicate $n=6)$; thus, 5 studies $[6,9,15-17]$ were included in the meta-analysis.

The basic characteristics of the 5 included studies are summarized in Table I. Two studies recruited at least 100 patients, with a range of 52 to 211. The mean patient age ranged from 62.2 to 71.5 years, and the majority of the patients were males with a range of $54 \%$ to $81 \%$ between studies. The number of involved limbs in which SPP was evaluated ranged from 29 to 123. In all studies, SPP was measured by the laser Doppler method.

\section{Sensitivity and specificity of SPP for wound healing}

Results of the meta-analysis of the sensitivity and specificity of SPP for the prediction of wound healing are shown in Figure 2. There was evidence of significant heterogeneity with respect to sensitivity across all 5 studies and when the 2 cutoff points were examined (all studies: $Q=10.63$, $p=0.031, I^{2}=62.4 \% ; 30 \mathrm{~mm}$ Hg cut-off: $Q=1.48$, $p=0.476, R^{2}=0 \% ; 40 \mathrm{~mm} \mathrm{Hg}$ cut-off: $Q=2.01$, $p=0.156, R^{2}=50.4 \%$ ); therefore, random-effects models of analysis were used. The pooled sensitivity of SPP for the prediction of wound healing was $79.9 \%$ for 3 studies using $30 \mathrm{~mm} \mathrm{Hg}$ as the cut-off, $67.1 \%$ for 2 studies using $40 \mathrm{~mm} \mathrm{Hg}$ as the cut-off, and $76.1 \%$ for all included studies $(95 \% \mathrm{Cl}$ : 73.9-84.9\%, 55.8-76.8\%, 70.7-80.8\%, respectively) (Figure $2 \mathrm{~A}$ ).

There was evidence of significant heterogeneity with respect to specificity across all 5 studies and when the 2 cut-off points were examined (all studies: $Q=15.81, p=0.003, I^{2}=74.7 \% ; 30 \mathrm{~mm} \mathrm{Hg}$ cut-off: $Q=8.44, p=0.015, R^{2}=76.3 \%$; $40 \mathrm{~mm} \mathrm{Hg}$ : $\left.Q=2.03, p=0.154, l^{2}=50.8 \%\right)$; therefore, random-effects models of analysis were used. The pooled specificity of SPP for the prediction of

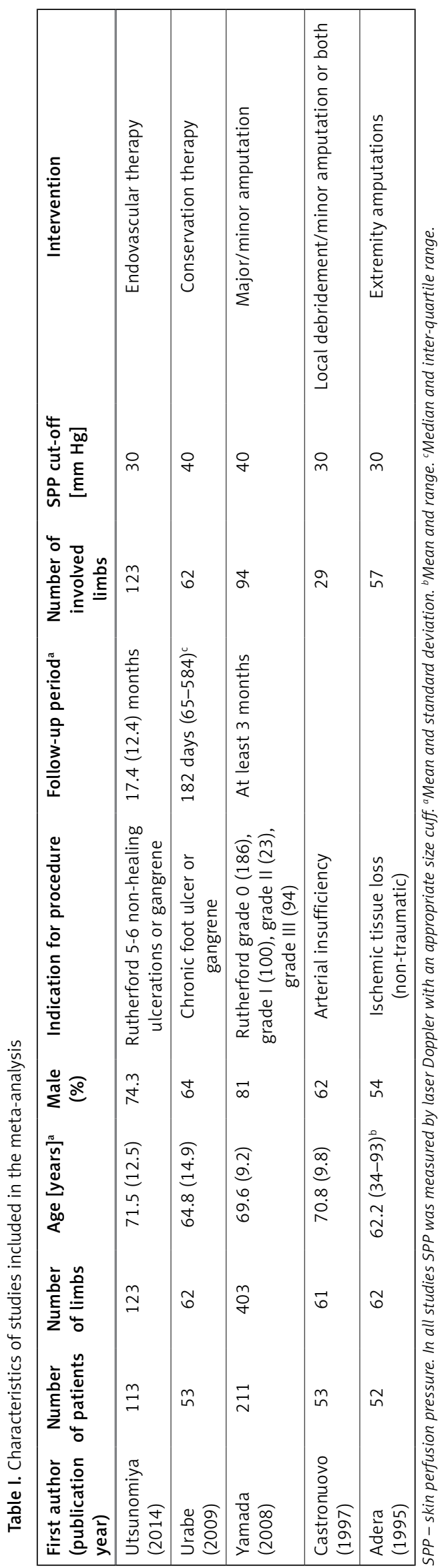


A Sensitivity

\begin{tabular}{|c|c|c|c|}
\hline \multirow[t]{2}{*}{ Cut-off } & \multirow[t]{2}{*}{ Study name } & \multicolumn{2}{|c|}{ Statistics for each study } \\
\hline & & Sensitivity & $95 \% \mathrm{Cl}$ \\
\hline \multirow[t]{3}{*}{$30 \mathrm{~mm} \mathrm{Hg}$} & Utsunomiya, 2014 & 0.814 & $0.735-0.873$ \\
\hline & Castronuovo, 1997 & 0.850 & $0.672-0.940$ \\
\hline & Adera, 1995 & 0.750 & $0.622-0.845$ \\
\hline \multicolumn{2}{|c|}{ Subtotal $\left(Q=1.48, p=0.476, R^{2}=0 \%\right)$} & 0.799 & $0.739-0.849$ \\
\hline \multirow[t]{2}{*}{$40 \mathrm{~mm} \mathrm{Hg}$} & Urabe, 2009 & 0.611 & $0.485-0.724$ \\
\hline & Yamada, 2008 & 0.720 & $0.621-0.801$ \\
\hline \multicolumn{2}{|c|}{ Subtotal $\left(Q=2.01, p=0.156, P^{2}=50.4 \%\right)$} & 0.671 & $0.558-0.768$ \\
\hline \multicolumn{2}{|c|}{ Total effect $\left(Q=10.63, p=0.031, I^{2}=62.4 \%\right)$} & 0.761 & $0.707-0.808$ \\
\hline
\end{tabular}

B specificity

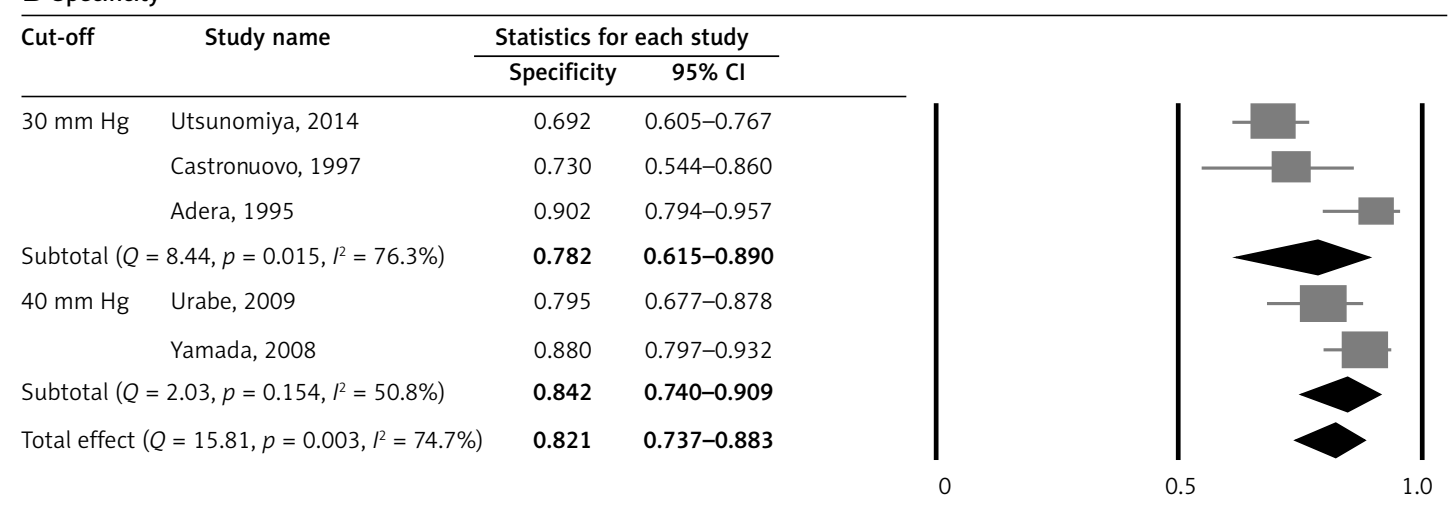

Figure 2. Meta-analysis of skin perfusion pressure for the prediction of wound healing: A - sensitivity, B-specificity

A Sensitivity

\begin{tabular}{lcc}
\hline Study name & \multicolumn{2}{c}{ Statistics with study removed } \\
\cline { 2 - 3 } & Sensitivity & $95 \% \mathrm{Cl}$ \\
\hline Utsunomiya, 2014 & 0.719 & $0.627-0.796$ \\
Urabe, 2009 & 0.774 & $0.714-0.824$ \\
Yamada, 2008 & 0.756 & $0.640-0.844$ \\
Castronuovo, 1997 & 0.731 & $0.639-0.806$ \\
Adera, 1995 & 0.747 & $0.637-0.833$ \\
Total effect & 0.746 & $0.661-0.816$
\end{tabular}

B specificity

\begin{tabular}{lcc}
\hline Study name & \multicolumn{2}{c}{ Statistics with study removed } \\
\cline { 2 - 3 } & Sensitivity & $95 \% \mathrm{Cl}$ \\
\hline Utsunomiya, 2014 & 0.837 & $0.754-0.896$ \\
Urabe, 2009 & 0.813 & $0.676-0.900$ \\
Yamada, 2008 & 0.781 & $0.670-0.863$ \\
Castronuovo, 1997 & 0.823 & $0.703-0.901$ \\
Adera, 1995 & 0.781 & $0.673-0.861$ \\
Total effect & 0.807 & $0.706-0.880$
\end{tabular}

Figure 3. Sensitivity analysis using the leave-one-out approach of skin perfusion pressure for the prediction of wound healing: A - sensitivity, B - specificity 
wound healing was $78.2 \%$ for 3 studies using the $30 \mathrm{~mm} \mathrm{Hg}$ cut-off, $84.2 \%$ for 2 studies using the $40 \mathrm{~mm} \mathrm{Hg}$ cut-off, and $82.1 \%$ for all included studies (95\% Cl: 61.5-89.0\%, 74.0-90.9\%, 73.7$88.3 \%$, respectively) (Figure $2 \mathrm{~B}$ ).

Results of the sensitivity analysis are shown in Figure 3. When each study was removed in turn, the sensitivity of SPP for the prediction of wound healing ranged from $71.9 \%$ to $77.4 \%$, and the specificity ranged from $78.1 \%$ to $83.7 \%$. These results indicate that individual studies did not influence the pooled estimates considerably.

Quality assessment using the QUADAS-2 instrument is shown in Figure 4. The results showed that the risk of bias and concerns regarding applicability were low, indicating that it is unlikely that the results of the meta-analysis are over- or underestimating the true intervention effect. However, retrospective studies were included in the meta-analysis, so there is potential selection and performance bias that may indirectly affect the validity of the analysis.

\section{Discussion}

The results of this meta-analysis, the first to study the accuracy of SPP for the prediction of wound healing, indicate that SPP provides adequate sensitivity and specificity for the prediction of wound healing in patients with critical limb ischemia. However, the sensitivity was better using a cut-off point of $30 \mathrm{~mm} \mathrm{Hg}$ as opposed to $40 \mathrm{~mm} \mathrm{Hg}$, while the specificity was better using a cut-off point of $40 \mathrm{~mm} \mathrm{Hg}$ as compared to $30 \mathrm{~mm} \mathrm{Hg}$. The cut-off values of SPP represent predictive values of successful wound healing prior to intervention. Although the evidence does not support that SPP can be used purely as a diagnostic tool, the results of our study showed that pre-procedural SPP for patients with Rutherford 5-6 limb ischemia may predict post-procedural wound healing.

Predicting the healing of ulcers and the need for amputation of ischemic limbs is challenging for physicians $[5,6]$. This is especially difficult in

A

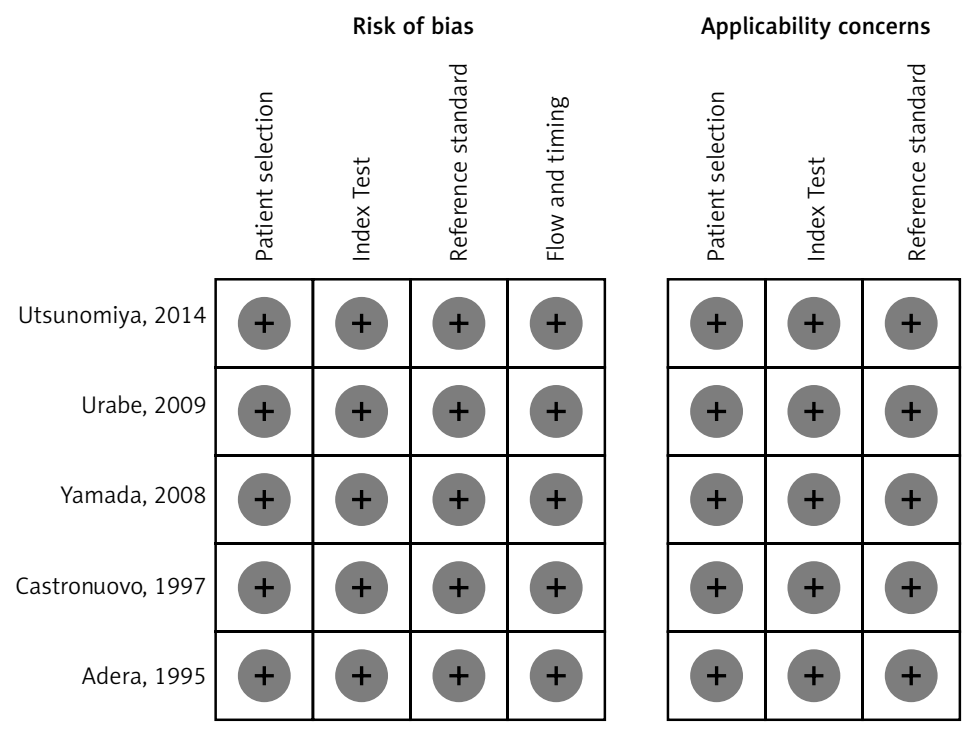

\begin{tabular}{|lll|}
\hline High Unclear $\quad+$ Low \\
\hline
\end{tabular}

B

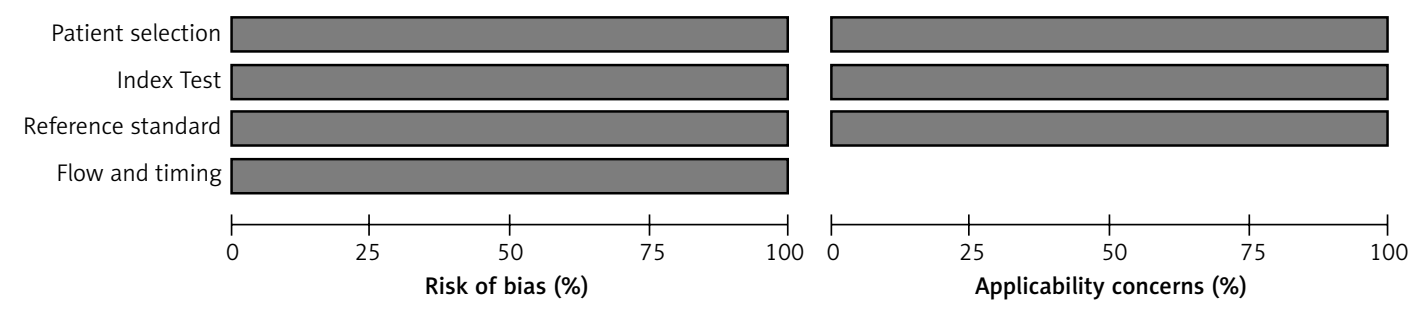

High

$\square$ Unclear

Low

Figure 4. Quality assessment of the included studies using the QUADAS-2 instrument: A - risk of bias summary,

B - overall assessment of risk of bias 
diabetic patients in whom arterial systolic ankle pressure in the affected limb measured by Doppler can be artificially elevated due to arterial calcifications of arteries [3]. Doppler ankle pressure has also been shown to have a poor predictive ability for the healing of forefoot amputations [18]. The measurement of SPP is a non-invasive method of determining the microcirculation in the limbs at approximately $1.5-2.0 \mathrm{~mm}$ beneath the skin [6]. Measurement of the SPP has been proven useful in assessment of ischemia severity, selecting amputation level, and for judging the likelihood that ischemic foot ulcers will heal $[6,7,15]$. While radioisotope clearance has generally been considered the "gold standard" for measuring SPP, Malvezzi et al. [8] showed a coefficient of correlation of 0.991 between radioisotope and laser Doppler methods of measuring SPP [8]. In this same study, 7 of 13 limbs tested demonstrated no clear deflection point using photoplethysmography, and thus an SPP reading could not be obtained.

Compared to radioisotope clearance, laser Doppler is a fast, simple, and efficient method, and is currently the most common method of measuring SPP $[6,7]$.

While SPP is a reliable tool, and is not influenced by arterial calcification, it has poor reproducibility and is influenced by factors such as skin and body temperature, sympathetic tone, vertical level of the limb, and possibly by edema and obesity. The findings of this study suggest that SPP is clinically valuable for assisting physicians in predicting wound healing, as well as assessing the degree of optimum amputation. However, current research has not identified a single perfect test for assessing perfusion in critical limb ischemia. A combination of tests such as SPP + hemodynamic measurements, or SPP + transcutaneous pressure of oxygen, may have better predictive results. Due to differences in optimum sensitivity and specificity cut-off values, physicians may adopt a middle ground of perhaps $35 \mathrm{~mm} \mathrm{Hg}$, or individualize the test results for each patient based on their clinical presentation.

The oldest study included in the current meta-analysis was published in 1995 and examined patients with above- and below-knee amputations [17]. The results showed that an SPP of $30 \mathrm{~mm} \mathrm{Hg}$ or greater predicted healing in $90 \%$ of cases, and a value below $30 \mathrm{~mm} \mathrm{Hg}$ predicted failure of healing in $75 \%$ of cases. In 1997, Castronuovo et al. [16] studied 61 limbs with nonhealing foot ulcers, and found that the overall diagnostic accuracy of SPP to diagnose critical limb ischemia was approximately $80 \%$. Of note, ankle-brachial index was not predictive of the need for reconstruction, major amputation, or the outcome of conservative local therapy. In 2008, Yamada et al.
[6] examined 403 limbs with arteriosclerosis obliterans of 211 patients, half of whom had diabetes or were receiving dialysis, and reported based on receiver operating characteristic (ROC) curve analysis that an SPP of $40 \mathrm{~mm} \mathrm{Hg}$ had a sensitivity of $72 \%$ and specificity of $88 \%$ for the prediction of wound healing. The authors also reported significant correlations between SPP and ankle blood pressure, toe blood pressure, and transcutaneous oxygen pressure. Urabe et al. [9] subsequently measured the SPP in 62 limbs in 53 patients and used a value of $40 \mathrm{~mm} \mathrm{Hg}$ for clinical decision making. Patients were treated with conservative therapy, and outcomes at 1 month were categorized as "improved" or "no change or worse". An SPP $\geq 40 \mathrm{~mm} \mathrm{Hg}$ predicted the 1-month outcome with a sensitivity of $75.0 \%$, specificity of $82.6 \%$, and an accuracy of $80.6 \%$. Logistic regression analysis showed that SPP $\geq 40 \mathrm{~mm} \mathrm{Hg}$ was an independent predictor of improved outcome with an odds ratio of $14.2(95 \% \mathrm{Cl} ; 3.6-55.8$, $p<0.0001)$. The most recently published study in the current analysis was reported by Utsunomiya et al. [15] in 2014. The authors examined 123 limbs in 113 patients who underwent successful balloon angioplasty with or without stenting, and ROC analysis indicated that the optimal SPP cutoff for predicting wound healing was $30 \mathrm{~mm} \mathrm{Hg}$, with a sensitivity of $81.4 \%$ and a specificity of $69.2 \%$. Logistic regression analysis showed that SPP was an independent predictor of wound healing, and the probability of wound healing with SPP values > $30 \mathrm{~mm} \mathrm{Hg}, 40 \mathrm{~mm} \mathrm{Hg}$, and $50 \mathrm{~mm} \mathrm{Hg}$ were $69.8 \%, 86.3 \%$, and $94.5 \%$, respectively.

Other studies that did not meet the inclusion criteria for the current analysis have also examined the predictive value of SPP for wound healing. Watanabe et al. [19] retrospectively examined 19 lower limbs in 18 patients who underwent arterial reconstruction for critical limb ischemia and found that an SPP > $30 \mathrm{~mm} \mathrm{Hg}$ was necessary for wound healing. Recently, Okamoto et al. [20] performed a subanalysis of the OLIVE registry of patients who received infrainguinal endovascular therapy for critical limb ischemia and reported that postprocedural SPP was significantly correlated with 1-year amputation-free survival, modified major adverse limb events, and wound healing. Kawasaki et al. [21] examined patient position (supine, lower limbs elevated, sitting, reclining bed elevation of $20^{\circ}$ ) and SPP of the lower limb in 10 healthy adults and 11 patients with critical limb ischemia. SPP was significantly higher in the sitting position than in the other 3 positions in patients of both groups.

There are a number of limitations to the current analysis. In its purest form a meta-analysis will only include RCTs. However, the number of 
studies examining this topic is very limited. For this reason we believe it is appropriate to include studies that are not RCTs in the analysis. Even including studies that were not RCTs, only 5 studies were available for inclusion; 3 of the 5 examined a cut-off of $30 \mathrm{~mm} \mathrm{Hg}$, and 2 of the 5 examined a cut-off of $40 \mathrm{~mm} \mathrm{Hg}$. The numbers of patients in the studies were also relatively small, and the treatment of patients also varied between debridement/conservative management, amputation, and endovascular treatment. Because of the limited data, the type of intervention could not be taken into account, and the timing of SPP measurement varied between the studies. For example, Utsunomiya et al. [15] measured SPP before and within 24-48 $\mathrm{h}$ after endovascular therapy, Yamada et al. [6] and Castronuova et al. [16] measured SPP at the proximal margin of the ulcer before amputation, Adera et al. [17] measured SPP before amputation, and Urabe et al. [9] measured SPP before conservative treatment. These limitations may affect the reliability of the results.

In conclusion, SPP is an accurate method for prediction of wound healing in patients with critical limb ischemia. Further study is necessary to determine whether a cut-off value of $30 \mathrm{~mm} \mathrm{Hg}$ or $40 \mathrm{~mm} \mathrm{Hg}$ provides the best diagnostic accuracy, as the limitations of this meta-analysis prevent us from drawing a robust conclusion or a "gold-standard" cut-off value of SPP for predicting wound healing.

\section{Conflict of interest}

The authors declare no conflict of interest.

\section{References}

1. Novo S, Coppola G, Milio G. Critical limb ischemia: definition and natural history. Curr Drug Targets Cardiovasc Haematol Disord 2004; 4: 219-25.

2. Skóra J, Pupka A, Janczak D, et al. Combined autologous bone marrow mononuclear cell and gene therapy as the last resort for patients with critical limb ischemia. Arch Med Sci 2015; 11: 325-31.

3. Karanfilian RG, Lynch TG, Zirul VT, Padberg FT, Jamil Z, Hobson RW 2nd. The value of laser Doppler velocimetry and transcutaneous oxygen tension determination in predicting healing of ischemic forefoot ulcerations and amputations in diabetic and nondiabetic patients. J Vasc Surg 1986; 4: 511-6.

4. Varga M, Sixta B, Bem R, Matia I, Jirkovska A, Adamec M. Application of gentamicin-collagen sponge shortened wound healing time after minor amputations in diabetic patients - a prospective, randomised trial. Arch Med Sci 2014; 10: 283-7.

5. Holstein P. Level selection in leg amputation for arterial occlusive disease: a comparison of clinical evaluation and skin perfusion pressure. Acta Orthop Scand 1982; 53: 821-31.

6. Yamada T, Ohta T, Ishibashi H, et al. Clinical reliability and utility of skin perfusion pressure measurement in ischemic limbs: comparison with other noninvasive diagnostic methods. J Vasc Surg 2008; 47: 318-23.

7. Lo T, Sample R, Moore P, Gold P. Prediction of wound healing outcome using skin perfusion pressure and transcutaneous oximetry: a single-center experience in 100 patients. Wounds 2009; 21: 310-6.

8. Malvezzi L, Castronuovo JJ Jr, Swayne LC, Cone D, Trivino JZ. The correlation between three methods of skin perfusion pressure measurement: radionuclide washout, laser Doppler flow, and photoplethysmography. J Vasc Surg 1992; 15: 823-9.

9. Urabe G, Yamamoto K, Onozuka A, Miyata T, Nagawa H. Skin perfusion pressure is a useful tool for evaluating outcome of ischemic foot ulcers with conservative therapy. Ann Vasc Dis 2009; 2: 21-6.

10. Liberati A, Altman DG, Tetzlaff J, et al. The PRISMA statement for reporting systematic reviews and meta-analyses of studies that evaluate health care interventions: explanation and elaboration. Ann Intern Med 2009; 151: W65-94.

11. Whiting PF, Rutjes AW, Westwood ME, et al. QUADAS-2: a revised tool for the quality assessment of diagnostic accuracy studies. Ann Intern Med 2011; 155: 529-36.

12. Lau J, loannidis JP, Schmid CH. Quantitative synthesis in systematic reviews. Ann Intern Med 1997; 127: 820-6.

13. Higgins JP, Thompson SG. Quantifying heterogeneity in a meta-analysis. Stat Med 2002; 21: 1539-58.

14. DerSimonian R, Laird N. Meta-analysis in clinical trials. Control Clin Trials 1986; 7: 177-88.

15. Utsunomiya $M$, Nakamura $M$, Nagashima $Y$, Sugi $K$. Predictive value of skin perfusion pressure after endovascular therapy for wound healing in critical limb ischemia. J Endovasc Ther 2014; 21: 662-70.

16. Castronuovo JJ Jr, Adera HM, Smiell JM, Price RM. Skin perfusion pressure measurement is valuable in the diagnosis of critical limb ischemia. J Vasc Surg 1997; 26: 629-37.

17. Adera HM, James K, Castronuovo JJ Jr, Byrne M, Deshmukh R, Lohr J. Prediction of amputation wound healing with skin perfusion pressure. J Vasc Surg 1995; 21 : 823-8.

18. Mehta K, Hobson RW 2nd, Jamil Z, Hart L, O’Donnell JA. Fallibility of Doppler ankle pressure in predicting healing of transmetatarsal amputation. J Surg Res 1980; 28: 466-70.

19. Watanabe Y, Onozuka A, Obitsu Y, et al. Skin perfusion pressure measurement to assess improvement in peripheral circulation after arterial reconstruction for critical limb ischemia. Ann Vasc Dis 2011; 4: 235-40.

20. Okamoto S, lida O, Nakamura M, et al. Postprocedural skin perfusion pressure correlates with clinical outcomes 1 year after endovascular therapy for patients with critical limb ischemia. Angiology 2015; 66: 862-6.

21. Kawasaki T, Uemura T, Matsuo K, et al. The effect of different positions on lower limbs skin perfusion pressure. Indian J Plast Surg 2013; 46: 508-12. 